\title{
Influence of Relays Location and Propagation Environment on the BER of Multiple Relay Systems
}

\section{Huda A. AL-Khafaji1, Haider M. AlSabbagh¹, Alauddin Al-Omary², and Hussain Al-Rizzo ${ }^{3}$}

${ }^{1}$ Department of Electrical Engineering, College of Engineering, University of Basra, Basra, Iraq ${ }^{2}$ Department of Computer Engineering, College of IT, University of Bahrain, Bahrain

${ }^{3}$ Department of Systems Engineering, College of Engineering and Information Technology, University of Arkansas at Little Rock, USA

\section{Abstract}

Relay techniques have been recently employed to mitigate errors induced during data transmission and improve system performance. Bit Error Rate (BER) is one key metric used for system performance assessment. This paper provides analytical

Corresponding Author:

Alauddin Al-Omary

aalomary@uob.edu.bh

Received: 18 September 2018

Accepted: 10 October 2018

Published: 15 October 2018

Publishing services provided by Knowledge

(c) Huda A. AL-Khafaji et al. This article is distributed under the

terms of the

Attribution License, which permits unrestricted use and redistribution provided that the original author and source are credited.

Selection and Peer-review under the responsibility of the Sustainability and Resilience Conference Committee.

\section{G OPEN ACCESS} formulation for the BER as a function of distance employing Binary Phase Shift Keying (BPSK) modulation. Impact of the location of the relays and effects of propagation environment are considered. Results reveal that the best location of relays is in the center between the source and destination. Also, increasing number of relays are very beneficial in reducing the error rate.

Keywords: BER, BPSK, Amplify and Forward (AF) relaying, relay position, path loss exponent, cooperative relaying.

\section{Introduction}

In recent years wireless communications systems have witnessed a significant growth in services, since subscribers are looking for high speed data transfer with guaranteed quality of services. Many technique exist to satisfy such demanding requirements. One of the most important technique is Multi Input Multi Output (MIMO) and relay systems. In terms of simplicity and cost, the relay approach is preferred [1]. This paper investigates the utilization of relay technique and assess the system performance in terms relays location. The basic idea behind relaying, is to assist the main link between source and destination by providing an additional link for transmitting the signals [2]. Relays with a single antenna are placed in different locations to transmit a copy of the transmitted signal to form "virtual" MIMO without additional antennas providing 
space diversity [3]. There are two distinguished types of relays: Amplify and Forward (AF), and Decode and Forward (DF) protocols. In AF, the relay simply amplifies the received signal and resend it without any further signal processing. The amplification process can be regarded as a multiplication with an amplification factor $G$. The main drawback in AF relay is the noise introduced which is also amplified along with the signal, particularly for low Signal-to-Noise Ratio (SNR). To overcome this drawback, it is commonly used in the high SNR region. DF relays, on the other hand, decode the signal before sending it to the receiver. It is comparatively more complex than the AF [4]. The focus of this paper, is on AF relay with relatively high level of SNR. In such cooperative system, the BER is a key metric for performance assessment of the cooperative relaying system. Sivakumar et al., [5] investigate the effect of location of relays on the system performance and how to reduce the amount of transmitted power. Li et al. [6] examined the impact of relay placement on the system Symbol Error Rate (SER) and outage probability using Binary Phase Shift Keying (BPSK) modulation. The authors of [7] presented a comparison between performance of the AF and DF in terms of the BER when BPSK modulation is used over Rayleigh fading channel. Cho et al. [8] studied the effects of relay location on SER for AF and DF relays employing multiple relays. The results show that it is preferred to place the relays on the same location rather than on different locations in order to reduce the SER. In [9] Aldhaibani et al. examined the effects of the location of relays for cell edge users. In [10], the authors investigated effects of the relay placement on the SER and capacity. Their results show that the best performance is achieved when the relays are located in the middle between the source and the destination and the worst is when it is located close to the destination. Recently, Khalil et al. [11] presented performance assessment for system in terms of BER for multiple AF relays arranged in parallel over Rayleigh fading channel. This paper provides analytical formulation for the BER in terms of distances between source, relay and destination with existence of direct path. The BER simulated for a different relay location with a specific path loss exponent. Also, investigating effect of path loss exponent for different propagation environments and with different relay locations on the BER of multiple AF relay.

The rest of the paper is organized as follows. In Section II, the system model of the proposed AF system is described. In Section III, numerical results are presented to validate the theoretical analysis. Finally, Section IV concludes the paper. 


\section{Impact of Relay Location on the BER}

Large-scale fading in wireless system occurs when the transmitted signal passes through large distances compared to the wavelength and obstacles causing shadowing and path loss. Influence of the propagation environment in addition to the loss of signal power as function to the propagation distance cause path loss, while shadowing is the variation of signal power as it is impeded by obstacles [12]. The path in which the signal travels between the source and destination fluctuates from Line of Sight (LoS) to that harshly obstructed by objects with Non Line of Sight (NLOS) [13]. The path loss exponent, $\alpha$, used to capture the effect of path loss with the distance is expressed as $d \alpha$ [12]. The characteristic of different prorogation environments is referred to as path loss exponent value [14]. Low value of $\alpha$ means availability of LoS, for instant in free space environment $\alpha=2$, while larger values of $\alpha$ implies the existence of NLOS due to obstructions [13]. For example, LoS environment is represented by $\alpha=3$ and 4 while NLoS environment represents by $\alpha=5$ [15].

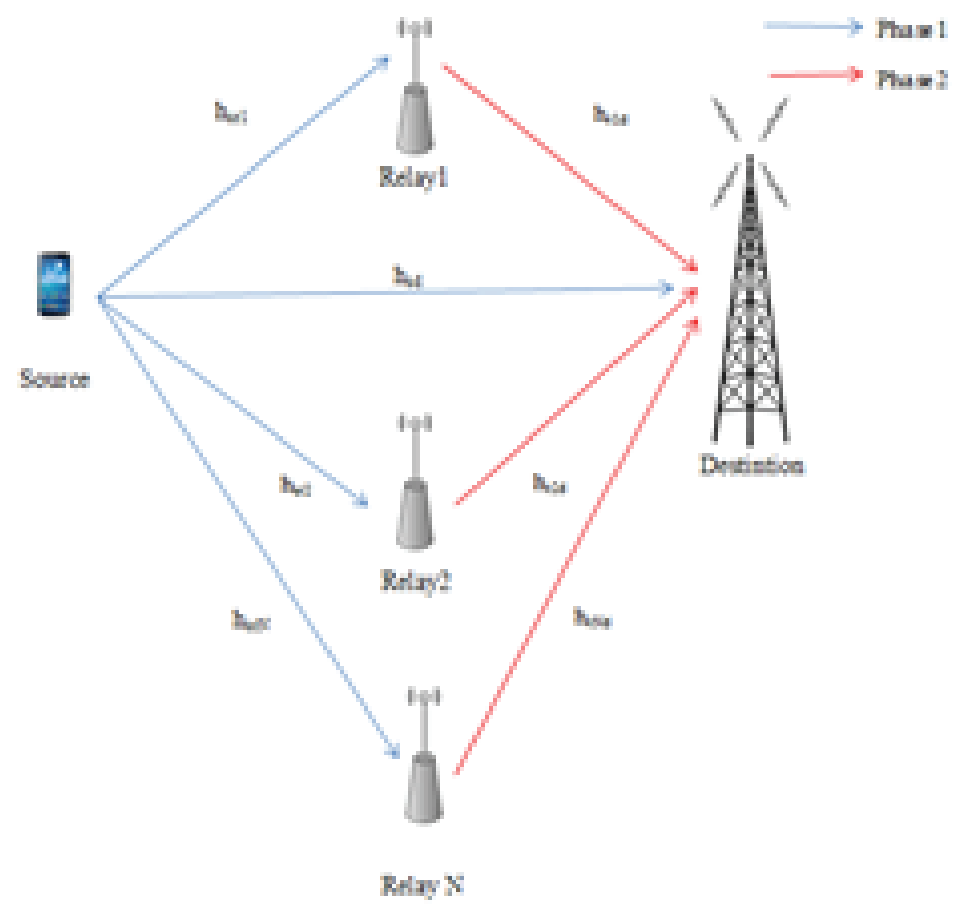

Figure 1: System model for cooperative diversity with direct link.

Considering the system shown in Fig.1, the signal from the source $s$ to the destination $\mathrm{d}$ is supported by $\mathrm{N}$ relays. The system works in a half-duplex transmission mode.

The transmission protocol requires two phases. In phase 1, the source broadcasts information to the destination, and the information at the same time naturally received 
by the relay. The received signal at the i-th relay node, ysri, and the destination, ysd, are given by [16]:

$$
\begin{gathered}
\text { ysri }=\sqrt{P_{t}} \text { hsrix }+ \text { nsri, } i=1,2,3 \ldots, N \\
\text { ysd }=\sqrt{P}_{t} \text { hsdx }+ \text { nsd },
\end{gathered}
$$

In phase $2, \mathrm{~N}$ relay nodes assist in amplifying the recieved signal received and then retransmit the signal to the destination node. The received signal at the destination due to the i-th relay transmission is:

$$
\text { yrid }=G_{r i} \text { hrid ysri + nrid }
$$

where $P_{t}$ denotes transmit power, $\mathrm{x}$ is the transmitted signal and $G_{r i}$ the amplification factor for i-th relay. The nsri, nsd and nrid are the additive white Gaussian noise (AWGN) of the source to destination link, the source to i-th relay link and i-th relay to the destination link, respectively with variance No. The hsd, hsri and hrid denote the channel coefficients modeled as a circularly symmetric complex Gaussian random variable with variances $\sigma_{s d}^{2}=d_{s d}{ }^{-\alpha}, \sigma_{s r i}^{2}=d_{s r i}{ }^{-\alpha}$ and $\sigma_{r i d}^{2}=d_{\text {rid }}{ }^{-\alpha}$, respectively [17]. $d_{s d}$, $d_{s r i}$ and $d_{r i d}$ are distances between the source and the destination nodes, source to $i$-th relay nodes and i-th relay to the destination nodes, respectively. In the simulations, the distance between the source and the destination is normalized as $d_{s d}=1 \mathrm{~km}$. Three cases are employed to simulate location of the relays: close to the source, close to the destination and in the center between the source and the destination. These three cases are investigated for availability of existing single and multi-relays. The values represent the location of the relay are assigned according to:

$$
d_{s d}=d_{s r i}+d_{r i d}
$$

the transmission gains are:

$$
h_{s r i}=\left(\frac{1}{d_{s r i}}\right)^{\frac{\alpha}{2}}, h_{r i d}=\left(\frac{1}{d_{r i d}}\right)^{\frac{\alpha}{2}} \text { and } h_{s d}=\left(\frac{1}{d_{s d}}\right)^{\frac{\alpha}{2}}
$$

the amplification factor of i-th relay Gri is [18]:

$$
G r i=\sqrt{\frac{P_{r i}}{P_{t} d_{s r i}{ }^{-\alpha}+N_{0}}}
$$

where Pri is the power at the $\mathrm{i}$-th relay. Let $\mathrm{Pt}=\mathrm{Ps}=\mathrm{Pri}$ and assume that the SNR of the source to destination link, $\gamma_{s d}$, the SNR of source to i-th relay link, $\gamma_{s r i}$, and SNR of the $\mathrm{i}$-th relay to the destination link, $\gamma_{\text {rid }}$, written in term of distances, as:

$$
\gamma_{\text {sri }}=\frac{P_{t} d_{\text {sri }}{ }^{-\alpha}}{N 0}, \gamma_{\text {rid }}=\frac{P_{t} d_{\text {rid }}^{-\alpha}}{N 0}
$$


and

$$
\gamma_{s d}=\frac{P_{t} d_{s d}^{-\alpha}}{N 0}
$$

The total SNR at the destination when a direct path link exists between the source and destination, is:

$$
\gamma_{d}=\sum_{i=1}^{N} \frac{\gamma_{s r i} \gamma_{r i d}}{\gamma_{s r i}+\gamma_{r i d}+1}+\gamma_{s d}
$$

For simplicity, assume SNR $=\frac{P_{t}}{N_{0}}$, the total SNR at the destination in Eq. 8 may be rewritten as:

$$
\gamma_{d}=\sum_{i=1}^{N} \frac{S N R^{2} d_{s r i}{ }^{-\alpha} d_{r i d}{ }^{-\alpha}}{S N R d_{s r i}{ }^{-\alpha}+S N R d_{r i d}{ }^{-\alpha}+1}+S N R d_{s d}{ }^{-\alpha}
$$

The BER for BPSK modulation is [19]:

$$
P_{b}=\frac{1}{2} \operatorname{erfc}\left(\sqrt{\gamma_{d}}\right)
$$

where erfc $(x)$ is the complementary error function.

\section{Simulation Results}

This section assesses the performance of the system in terms of BER using multiple AF relays at different locations in different propagation environments. Firstly, the effect of relay location with different path loss environments is discussed. Three different locations of the relay will be considered: relay at the center of the link (dsr1 $=0.5 \mathrm{~km})$, relay close to the source $(\mathrm{ds} r 1=0.3 \mathrm{~km})$ and relay close to the destination (dsr1 $=0.7$ $\mathrm{km})$. Eq. 4 is based for assigning all the distance values.

Figure 2 shows the impact of relay location on BER for multiple AF relays in suburban environment, $\alpha=4$ using BPSK modulation where all relays are located at the center of the link between the source and the destination. It is obvious that increasing the number of relays reduces the BER with increasing level of SNR. Variation of the BER with three relays when all are located close to the source and close to the destination, shown in Fig. 3 and Fig. 4, respectively. Similar performance is obtained with placing the relays close to the source or close to the destination. However, comparing the results in Figs. 2 and 3 against that in Fig. 4 reveals that the best location is at the center of the link between the source to the destination for all SNR. Figure 5 shows the BER for a single relay as a function of distance for different environments. From Fig. 5 (a), when the propagation is through sub-urban environment with LoS, $\alpha=3$, 


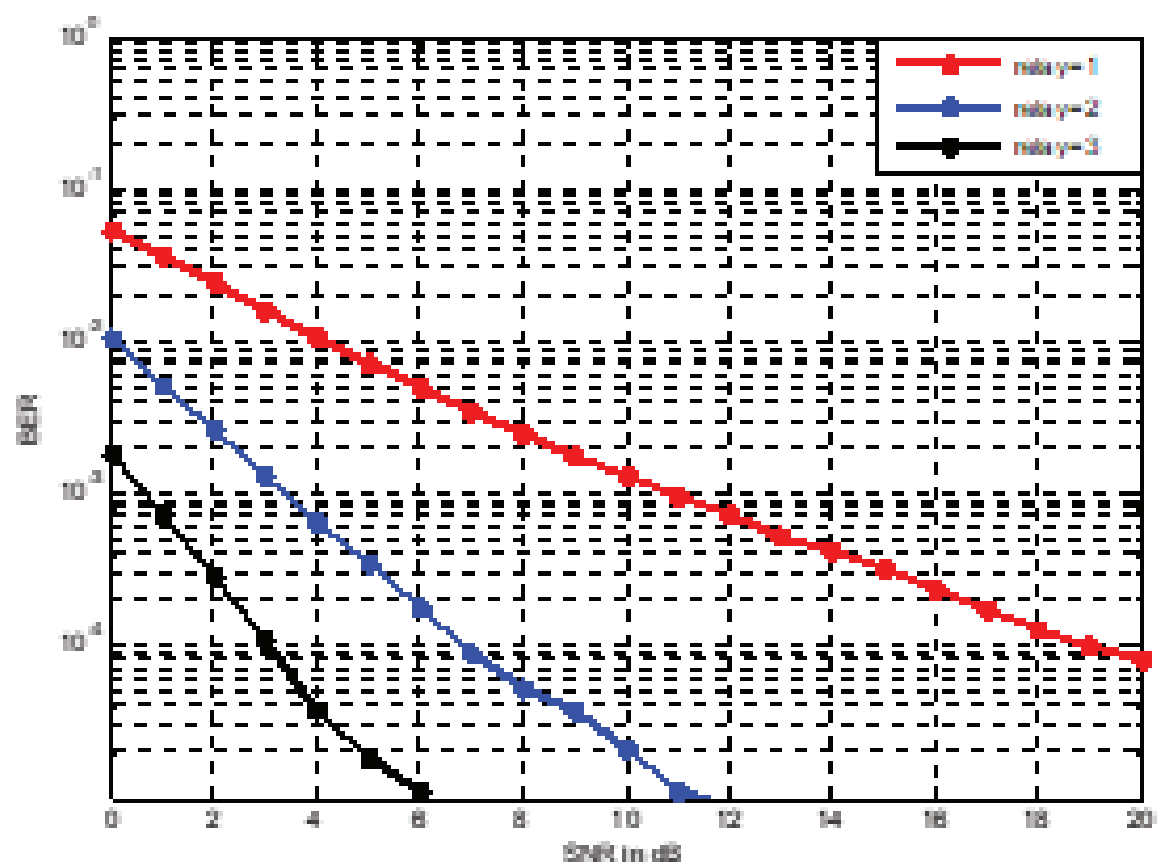

Figure 2: BER versus SNR for three cases: a single relay in red, two relays in blue, and three relays in black in all cases, the relays are at the center.

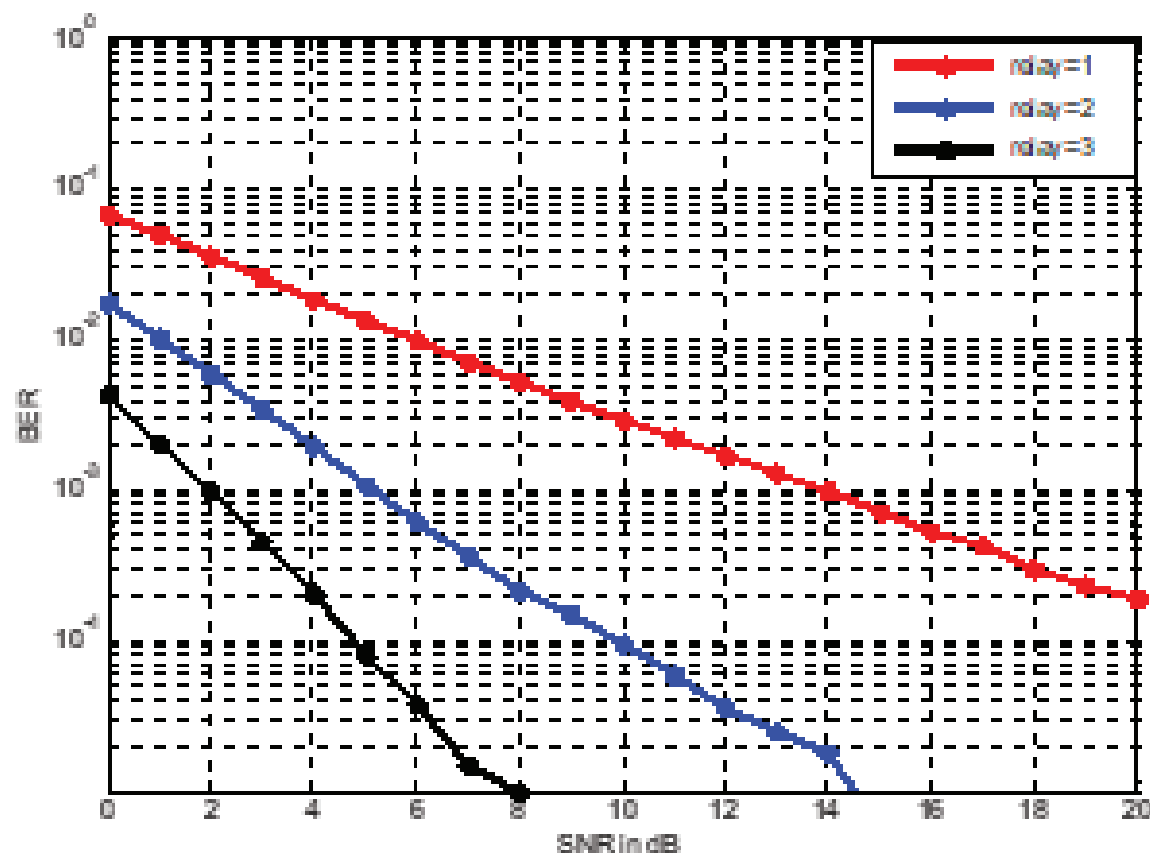

Figure 3: BER versus SNR for three cases: a single relay in red, two relays in blue and three relays in black in all cases, the three relays are close to the source.

the worst performance is when dsr $1=0.3$. The best performance is observed when $\mathrm{ds} r 1=0.7$ and SNR $>7 \mathrm{~dB}$. When $\alpha=4$ the availability of LoS decreases, the results show a small change of BER values in general. When $S N R \leq 10 \mathrm{~dB}$, the BER value of the $d s r 1=0.5$ has a lower value than that when the $d s r 1=0.7$ and they are almost 


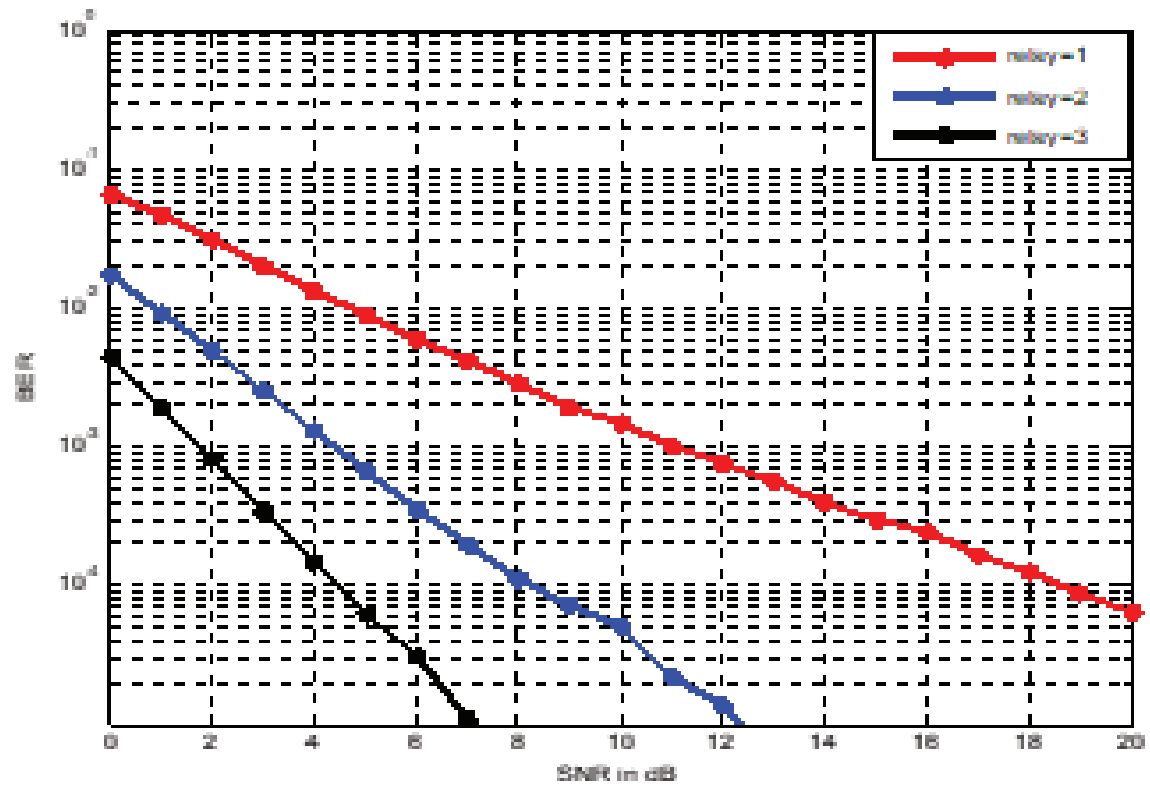

Figure 4: BER versus SNR for three cases: a single relay in red, two relays in blue and three relays in black in all cases, the three relays are close to the destination.

the same when the SNR is increased. When the dsr1 $=0.3$ and for all ranges of SNR, the BER value increased. When the signal propagates through harsh environment, $\alpha$ increased to 5, there is a significant change in BER values for all ranges of SNR and the best performance is obtained when the relay is in the center providing the lowest BER. This strategy of arranging of relays is preferred in "harsh" environment with large path loss exponent. The results for two relays are listed in Table 1 which presents results of employing two relays when the first relay is placed at the center while the second relay placed in: the center (dsr2 $=0.5)$, closer to the source $(\mathrm{dsr} 2=0.3$ ) and closer to the destination $(\mathrm{ds} r 2=0.7)$, respectively.

TABLE 1: The BER for two relays at SNR $=4 \mathrm{~dB}$

\begin{tabular}{|c|c|c|c|}
\hline$\alpha$ & BER when dsr1=0.5 & BER when dsr1=0.3 & BER when dsr1=0.7 \\
\hline 3 & $2.6 \times 10-3$ & $3.4 \times 10-3$ & $2.9 \times 10-3$ \\
\hline 4 & $6.6 \times 10-4$ & $1 \times 10-3$ & $1 \times 10-3$ \\
\hline 5 & $1.5 \times 10-4$ & $4 \times 10-4$ & $3 \times 10-4$
\end{tabular}

As $\alpha$ increases, the BER value decreases. As the propagation environment gets more dense, $\alpha$ increases, the values of the BER decrease, when $\alpha=5$, the best performance is obtained when $d s_{2}=0.5$ and the worst performance when ds $r 2=0.3$. Table 2 presents the results for three relays when the first and second relays are placed at the center and the third one is placed at a different locations: at the center $(\mathrm{ds} r 3=0.5)$, closer to the source $\left(\mathrm{ds} \mathrm{r}_{3}=0.3\right)$ and closer to the destination $(\mathrm{ds} r 3=0.7)$, respectively. 


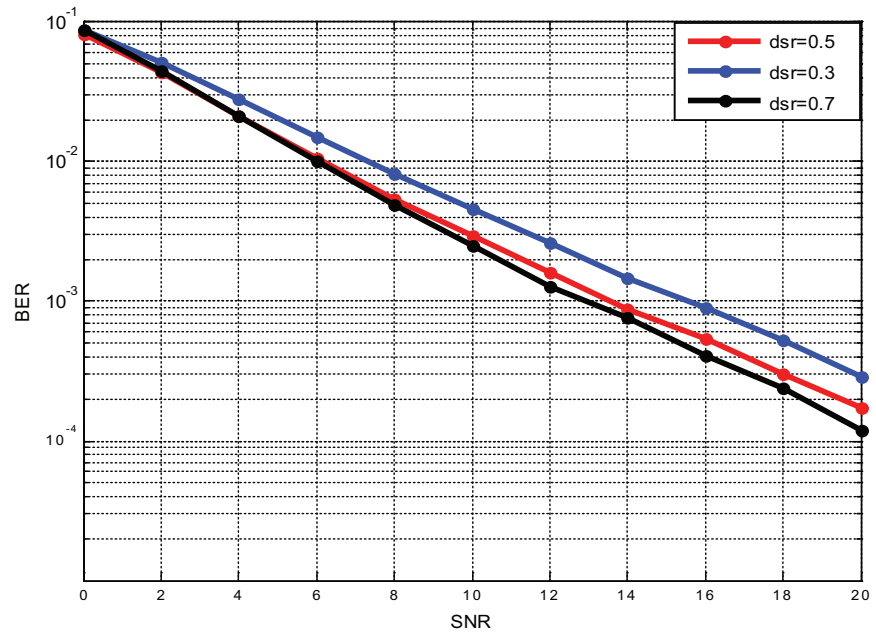

(a)

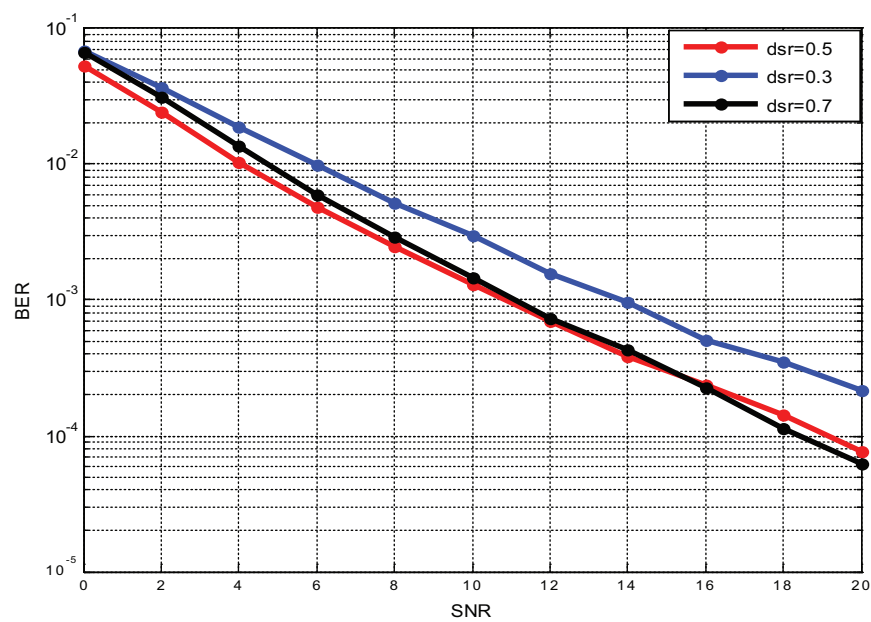

(b)

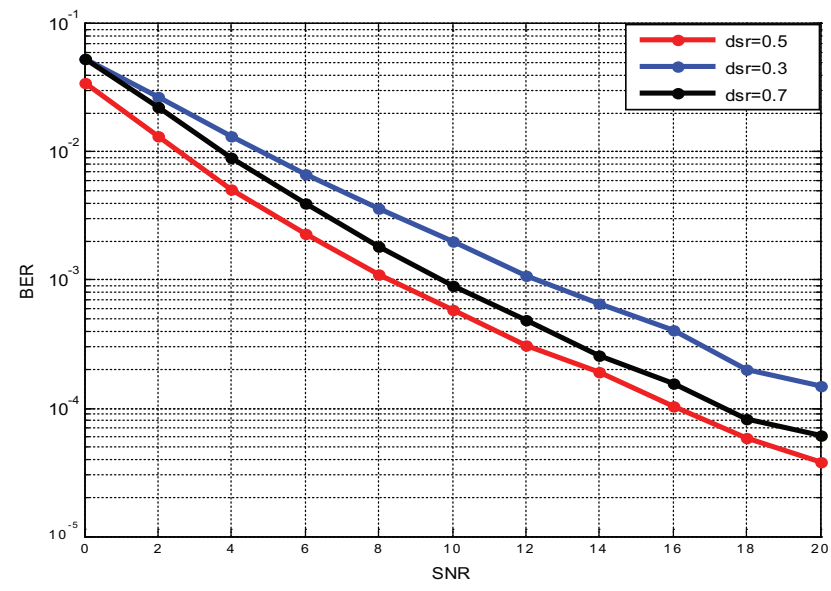

(c)

Figure 5: BER for a single relay for different propagation environments: (a) $\alpha=3$, (b) $\alpha=4$ and (c) $\alpha=5$. 
TABLE 2: The BER for three relays at SNR $=4 \mathrm{~dB}$.

\begin{tabular}{|c|c|c|c|}
\hline$\alpha$ & BER when dsr2 $=0.5$ & BER when dsr2 $=0.3$ & BER when dsr2 $=0.7$ \\
\hline 3 & $3.5 \times 10-4$ & $4 \times 10-4$ & $3.7 \times 10-4$ \\
\hline 4 & $5 \times 10-5$ & $7 \times 10-5$ & $7 \times 10-5$ \\
\hline 5 & $4 \times 10-6$ & $1.4 \times 10-5$ & $8 \times 10-6$
\end{tabular}

Increasing the number of relays generally decreases the BER and further it decreases more when the relay is located at the center. The reason behind the reduction in BER is that path loss is function to distance and since placing relays will split the distance between source and destination (dsd) into smaller distances. The BER in Eq.10 which is function to $d^{-\alpha}$ from $\gamma_{d}$, will associate with inverse relationships with $\alpha$.

\section{Conclusions}

In this paper, the BER for multiple AF relays in terms of location between the source and destination assuming direct path propagation is investigated. It also reports the effect of the path loss for different propagation environments with different number of relays at different locations. The simulation results show that the best performance with lowest BER value may be obtained when the relays are placed at the center of the link between the source and the destination. The results show that the propagation environment has an effect on BER in which higher path loss exponent (i.e., harsh propagation environment) results in low BER if the relay exists in the system. Also, the results show that increasing numbers of relays decrease the BER in the system. The lowest BER is $4 \times 10-6$ when the number of relays in the system are three and all placed at the center of the link when the signal propagate in NLOS environment with $\alpha$ equal to 5 .

\section{References}

[1] J. Wei, "Device-to-Device Based Cooperative Relaying for 5G Network: A Comparative Review," ZTE Communications, vol. 1, No. S1, pp. 60-66 , (2017).

[2] A. Abrol and R. K. Jha, "Power optimization in 5 G networks: A step towards GrEEn communication," IEEE Access, vol. 4, Pp. 1355-1374, (2016).

[3] N. Kapucu, M. Bilim, and I. Develi, "SER performance of amplify-and-forward cooperative diversity over asymmetric fading channels," Wireless personal communications, vol. 73, pp. 1117-1127, (2013). 
[4] M. I. Khalil, S. M. Berber, and K. W. Sowerby, "HighSNR approximation for performance analysis of two-way multiple relay networks," Physical Communication, Vol 24, pp. 62-72, (2017).

[5] V. V. Sivakumar, D. Hu, and P. Agrawal, "Relay positioning for energy saving in cooperative networks," in System Theory (SSST), 2013 45th Southeastern Symposium on, pp. 124-128, (2013).

[6] . Li, Y. Kun, M. Zhou, J. Wu, L. Song, Y. Li, et al., "Full-duplex Amplify-and-Forward Relaying: Power and Location Optimization," IEEE Transactions on Vehicular Technology, Vol 66, pp. 8458-8468, (2017).

[7] X. Liu and W. Du, "BER-Based Comparison between AF and DF in Three-Terminal Relay Cooperative Communication with BPSK Modulation," in Mobile Ad-Hoc and Sensor Networks (MSN), 2016 12th International Conference on, pp. 296-300, (2016).

[8] W. Cho, H. S. Oh, and D. Y. Kwak, "Effect of relay locations in cooperative networks," in Wireless Communication, Vehicular Technology, Information Theory and Aerospace \& Electronic Systems Technology, pp. 737-741, (2009).

[9] J. A. Aldhaibani, R. Ahmad, A. Yahya, and S. A. Azeez, "Increasing the coverage area through relay node deployment in long term evolution advanced cellular networks," in AIP Conference Proceedings, p. 070059, (2015).

[10] K. K. Gurrala and S. Das, "Impact of relay location on the performance of multirelay cooperative communication," International Journal of Computer Networks and Wireless Communications, vol. 2, pp. 226-231, (2012).

[11] M. I. Khalil, S. M. Berber, and K. W. Sowerby, "Bit error rate performance analysis in amplify-and-forward relay networks," Wireless networks, vol. 23, pp. 947-957, (2017).

[12] A. Goldsmith, Wireless communications: Cambridge university press, (2005)

[13] T. S. Rappaport, Wireless communications: principles and practice vol. 2: Prentice Hall PTR New Jersey, (1996).

[14] K. R. Liu, Cooperative communications and networking: Cambridge university press, (2009).

[15] J. R. Hampton, Introduction to MIMO communications: Cambridge university press, (2013).

[16] K. G. Seddik, A. K. Sadek, W. Su, and K. R. Liu, "Outage analysis of multi-node amplify-and-forward relay networks," in Wireless Communications and Networking Conference, 2006. WCNC 2006. IEEE, PP. 1184-1188, (2006). 
[17] P. Herhold, W. Rave, and G. Fettweis, "Relaying in cdma networks: Pathloss reduction and transmit power savings," in Vehicular Technology Conference, 2003. VTC 2003-Spring. The 57th IEEE Semiannual, pp. 2047-2051, (2003).

[18] M. Wu, D. Wübben, and A. Dekorsy, "BER-based power allocation for amplify-andforward and decode-and-forward relaying systems," in Smart Antennas (WSA), 2011 International ITG Workshop on, Pp. 1-8, (2011).

[19] M. Wu, D. Wübben, and A. Dekorsy, "BER-based power allocation for decodeand-forward relaying with m-qam constellations," in Wireless Communications and Mobile Computing Conference (IWCMC) 7th International, pp. 888-893, (2011). 